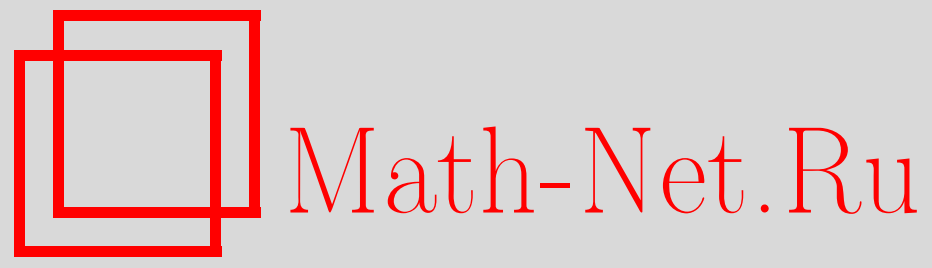

Т. А. Белкина, В. И. Ротарь, Об оптимальности по вероятности и почти наверное для процессов со свойством связности. І. Случай дискретного времени, Теория вероятн. и ее примен., 2005, том 50, выпуск 1, 3-26

DOI: https://doi.org/10.4213/tvp156

Использование Общероссийского математического портала MathNet.Ru подразумевает, что вы прочитали и согласны с пользовательским соглашением http://www . mathnet.ru/rus/agreement

Параметры загрузки:

IP : 54.205 .225 .156

26 апреля 2023 г., 06:51:59

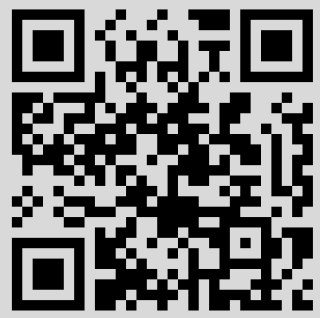




\title{
ОБ ОПТИМАЛЬНОСТИ ПО ВЕРОЯТНОСТИ И ПОЧТИ НАВЕРНОЕ ДЛЯ ПРОЦЕССОВ СО СВОЙСТВОМ СВЯЗНОСТИ. І. СЛУЧАЙ ДИСКРЕТНОГО ВРЕМЕНИ ${ }^{1)}$
}

\begin{abstract}
Получены условия, при которых стратегии, минимизирующие математическое ожидание целевого функционала (оптимальные в среднем), обладают более сильным свойством, а именно, минимизируют значение самого функционала для всех реализаций управляемого случайного процесса из множества, вероятность которого близка к единице при больших горизонтах планирования. Главное отличие указанных условий от полученных ранее состоит в том, что они связаны не со свойствами функции Беллмана, а с возможностью перевода управляемого процесса из одного состояния в другое за время с конечным математическим ожиданием. Это делает проще проверку условий в целом ряде ситуаций общего характера. Первая часть статьи касается процессов с дискретным временем, вторая - с непрерывным временем.

Kлючевые слова и фразы: управляемые процессы, управляемые марковские цепи, асимптотическая оптимальность, оптимальность почти наверное, оптимальность по вероятности.
\end{abstract}

\section{$\S 1$ Введение и результаты для некоторых простых моделей}

1.1. Введение. В задачах стохастического динамического управления с конечным горизонтом планирования оптимальность традиционно понимается в смысле минимизации по всем допустимым управлениям математического ожидания целевого функционала. В случае, когда на каждом конечном интервале времени задан аддитивный целевой функционал, а поведение системы необходимо исследовать на бесконечном интервале, используется нормировка функционала делением

* Центральный экономико-математический институт РАН, Нахимовский пр-т, 47, 117418 Москва, Россия; e-mail: tbel@cemi.rssi.ru

** Department of Mathematics and Statistics of San Diego State University, San Diego, CA 92182-7720, USA; e-mail: rotar@sciences.sdsu.edu

1) Работа выполнена при частичной поддержке Российского фонда фундаментальных исследований (грант № 03-01-00479) и РАН по итогам 6-го конкурса-экспертизы проектов молодых ученых (грант № 105). 
на длину интервала планирования, не меняющая задачи оптимизации. Тогда соответствующий традиционный подход связан с исследованием асимптотического поведения математического ожидания среднего (в единицу времени) значения функционала. В дальнейшем экстремальные управления в задачах при подобных постановках будем называть управлениями, оптимальными в среднем (average optimal).

В последние годы повысился интерес к сравнительно новому направлению в стохастической оптимизации, связанному с более сильным понятием оптимальности. В контексте этого направления исследуются управления, минимизирующие не только математическое ожидание целевого функционала, но и значение самого функционала с вероятностью, асимптотически близкой к единице при больших горизонтах планирования. Как правило, если такие управления существуют, среди них имеются также оптимальные в среднем.

Насколько нам известно, первые результаты в этом направлении были получены в [22] и [30], где рассматривались рекуррентные полумарковские процессы. Существенное продвижение было достигнуто в [32]-[37]; см. там же ссылки на другие статьи тех же авторов. В этих работах рассматривались управляемые марковские цепи и впервые сделана попытка применения мартингального подхода для решения указанной проблемы. Позже в [44] изучалась задача на бесконечном интервале времени для марковских управляемых процессов в более общей постановке. В [28], [9]-[13] исследовались с этих позиций однородные диффузионные процессы. В частности, для этого случая в [9] было введено в рассмотрение понятие устойчивого стационарного управления.

Общая схема динамической оптимизации с дискретным временем с соответствующими определениями оптимальности почти наверное и по вероятности была рассмотрена в [41], [2]. Общая постановка для диффузионных процессов была предложена в [38].

В то время как исследование данной проблемы в рамках общих постановок, описанных в упомянутых работах, оказывается достаточно сложным, некоторые частные схемы изучены более детально. В основном это касается линейной модели с квадратичным функционалом (линейного регулятора); см., например, [14] для ARMAX-модели, [33], [20, гл. 7], [27], [5], [25] для линейного регуंлятора с дискретным временем, и [38], [7] для линейного регулятора с непрерывным временем. Дальнейшие ссылки и более подробные комментарии см. также в разделе 1.3.

Следует заметить, что определения асимптотической оптимальности иногда различны (и не эквивалентны) в различных работах; подробнее см. раздел 1.2. Мы будем следовать определениям оптимальности по вероятности и почти наверное, введенным в [41] и [2].

Общие условия, приводимые в упомянутых выше статьях, касаются свойств функции Беллмана для задачи минимизации ожидаемого зна- 
чения целевого функционала. Эти условия заключаются в требовании того, чтобы функция Беллмана не слишком сильно зависела от начального состояния процесса, так же как и от решений, принимаемых на начальных шагах управления. Точнее, влияние указанных факторов должно выражаться только членами второго порядка в асимптотическом представлении функции Беллмана при больших горизонтах планирования. Это означает, в частности, что для того, чтобы проверить эти условия, нужно сначала найти оптимальное управление и - более или менее точно - саму функцию Беллмана. Но это далеко не всегда возможно.

В данной работе мы рассматриваем предположения другого типа, связанные со свойством связности процесса. Простейшие предположения такого типа могут быть качественно описаны следуюшим образом.

Рассмотрим траекторию, начинающуюся из фиксированного начального состояния и порожденную оптимальной в среднем стратегией. Выберем другое начальное состояние и рассмотрим такие стратегии, что порожденные ими траектории, начинающиеся из этого состояния, пересекают первую траекторию за некоторое (случайное) время. Тогда условие состоит в том, что среди этих стратегий существует такая, для которой ожидаемое значение указанного времени слияния траекторий ограничено равномерно по всем начальным состояниям.

Главная идея здесь состоит в том, что для того, чтобы проверить эти условия, не нужно знать ни точное представление стратегии, оптимальной в среднем, ни функцию Беллмана. Например, достаточно проверить возможность перехода из одного состояния в другое за «не слишком большое время». Простейшим примером здесь может служить конечная управляемая марковская цепь. В этом случае обсуждаемое свойство связности выполнено, если существует допустимая стратегия (возможно, очень «плохая》), при которой процесс переводится из одного состояния в другое, скажем, с равными вероятностями. Еще одним простым примером может служить линейная система с произвольной ограниченной ценой управления на одном шаге: в этом случае проверка упомянутых условий также проста. Ниже будут приведены примеры, показывающие, что то же верно в значительно более общих нелинейных моделях.

Таким образом, когда обсуждаемые условия проверены, но не представляется возможности найти точное представление оптимальной в среднем стратегии, можно проделать это численно и при этом быть уверенными в том, что эта стратегия оптимальна не только в среднем, но и асимптотически почти наверное.

Для случая дискретного времени в менее общей ситуации (по сравнению с рассматриваемой в данной работе) результаты в этом направлении были получены в [42]. 
В части I этой статьи мы изучаем случай дискретного времени; процессы с непрерывным временем рассматриваются в части II, которая будет опубликована позднее.

Общая ситуация, в рамках которой формулируется основная теорема, будет исследоваться в $\S 2$. В заключение этого параграфы рассмотрим некоторые более простые условия и следствия из основной теоремы. Все доказательства приводятся в $\S 3$.

1.2. Марковская цепь с дискретным временем: оптимальность почти наверное.

1.2.1. Определение оптимальности. Определим управляемую однородную марковскую цепь $x_{t}, t=0,1,2, \ldots$, со значениями в $\mathbf{R}^{l}$ следующим рекуррентным соотношением:

$$
x_{t}=h\left(\xi_{t}, x_{t-1}, a_{t}\right)
$$

где $\xi_{1}, \xi_{2}, \ldots$ - независимые одинаково распределенные (н.о.р.) случайные величины (с.в.), $a_{t}$ - элемент пространства $\mathbf{R}^{m}$ и $h: \mathbf{R} \times \mathbf{R}^{l} \times$ $\mathbf{R}^{m} \rightarrow \mathbf{R}^{l}$ - некоторая измеримая функция. Будем интерпретировать $a_{t}$ как решение, принимаемое в момент $t$.

Ограничимся евклидовым пространством только в целях упрощения некоторых определений и доказательств. В 2 будут рассматриваться пространства общей природы.

Заметим также, что при другом подходе управляемая марковская цепь определяется с помощью переходной меры $Q(\cdot \mid x, a)$ - условного распределения $x_{t}$ при заданных $x_{t-1}=x, a_{t}=a$. С другой стороны, хорошо известно, что эти подходы эквивалентны, в частности, для каждой $Q$ существует функция $h$ такая, что распределение с.в. $h\left(\xi_{t}, x, a\right)$ совпадает с $Q(\cdot \mid x, a)$. Более того, с.в. $\xi$ можно считать равномерно распределенными на интервале [0,1]; см., например, [19]. Однако представление (1.1) более удобно для наших целей, так как существование единого вероятностного пространства позволяет сравнивать процессы, порожденные различными стратегиями.

Ниже для любой последовательности элементов $\alpha_{1}, \alpha_{2}, \ldots$ положим $\alpha^{t}=\left(\alpha_{1}, \ldots, \alpha_{t}\right)$.

Пусть $x_{0}=x$. Для любого целого $n \geqslant 1$ рассмотрим функционал

$$
J_{x n}\left(a^{n}\right)=\sum_{t=1}^{n} q\left(x_{t}, a_{t}\right),
$$

где $x_{t}$ удовлетворяет (1.1) при $x_{0}=x$, а измеримая функция $q$ определяет цену управления в единицу времени.

Чтобы определить управление, положим $a_{t}=u_{t}$, где $u_{t}-$ случайная величина, измеримая относительно $\sigma$-алгебры $\mathscr{F}_{t-1}=\sigma\left\{\xi_{1}, \ldots, \xi_{t-1}\right\}$. С.в. $u_{t}$ представляет собой управление в момент $t$. 
Символом $u$ будем обозначать бесконечную последовательность $\left\{u_{1}, u_{2}, \ldots\right\}$, символом $u^{n}$ - ее сужение $\left\{u_{1}, \ldots, u_{n}\right\} . \mathrm{K}$ последовательности $u$ также будем применять термин «управление». Запись $J_{x n}(u)$ будет означать $J_{x n}\left(u^{n}\right)$.

Заметим, что формально данное выше определение фильтрации $\left\{\mathscr{F}_{t}\right\}$ предполагает более богатую структуру информации, чем в некоторых стандартных моделях: так как $u_{t}$ измерима относительно $\mathscr{F}_{t-1}$, мы наблюдаем случайные величины $\xi$, а не состояния $x$. Но так как функционал (1.2) определяется значениями $x_{t}$ и $a_{t}$, в конечном счете мы можем ограничиться достаточными управлениями, измеримыми относительно фильтрации, порожденной последовательностью $x_{t}$, т.е. управлениями, порожденными стратегиями (см. п. 1.2.2; о достаточных управлениях см., например, [40]). Тем не менее, чтобы избежать некоторых громоздких конструкций, мы будем использовать приведенное выше определение управления.

В множестве всевозможных управлений выделим некоторый, вообще говоря, произвольный класс $\mathscr{U}$; управления из этого класса будем называть допустимыми. В качестве класса допустимых управлений в некоторых случаях может рассматриваться наиболее широкий класс всевозможных управлений, т.е. бесконечных последовательностей $u$ с описанными выше свойствами. В другом случае класс $\mathscr{U}$ может быть более узким, например, он может состоять только из управлений, порожденных стратегиями (строгое определение см. ниже).

Для $n \geqslant 1$ интервал времени $\{t ; t=0,1, \ldots, n\}$ будем обозначать $\{0, n\}$. Управление $\widehat{u}^{n}$ называется оптимальныл в среднем (average optimal) на интервале $\{0, n\}$ при начальном состоянии $x$, если

$$
\mathbf{E} J_{x n}\left(\widehat{u}^{n}\right)=\inf _{u^{n}} \mathbf{E} J_{x n}\left(u^{n}\right),
$$

где инфимум берется по всем сужениям $u^{n}$ управлений из $\mathscr{U}$.

Ясно, что $\widehat{u}^{n}$, если оно существует, может зависеть от $x$ и $n$, так что, рассматривая последовательность $\left\{\widehat{u}^{n}\right\}$, мы имеем дело со схемой серий $\left\{\widehat{u}_{1 n}, \ldots, \widehat{u}_{n n}\right\}$. Позже мы будем рассматривать общий случай, когда управление характеризуется схемой серий $\left\{u^{n}=\left(u_{1 n}, \ldots, u_{n n}\right)\right\}$; в этом параграфе мы ограничимся традиционной постановкой, включающей только бесконечные последовательности управлений $\left\{u_{1}, u_{2}, \ldots\right\}$.

О п р е д е л е н и е 1 . Для данного начального состояния $x$ управление $u^{*} \in \mathscr{U}$ называется асимптотически оптимальным почти наверное, если для любого управления $u \in \mathscr{U}$

$$
\liminf _{n \rightarrow \infty}\left[n^{-1}\left(J_{x n}(u)-J_{x n}\left(u^{*}\right)\right)\right] \geqslant 0 \quad \text { п.н. }
$$

Данное определение не исчерпывает всех известных определений такого типа. Одна из хорошо известных схем (см., например, [32], [33], [20] 
для линейного регулятора, [12] для общей стационарной модели, [21] для некоторых моделей в дискретном времени, а также ссылки в упомянутых работах) связана с определением, при котором

(а) существуют управление $\bar{u}$ и число $\theta$ такие, что

$$
\lim _{n \rightarrow \infty} n^{-1} J_{x n}(\bar{u})=\theta \quad \text { п.н.; }
$$

(b) для любого допустимого управления $u$

$$
\limsup _{n \rightarrow \infty} n^{-1} J_{x n}(u) \geqslant \theta \quad \text { п.н. }
$$

По нашему мнению, свойство оптимальности в последнем смысле менее предпочтительно, чем данное в (1.3), по двум соображениям. Вопервых, (1.3) не предполагает выполнения закона больших чисел (как в (1.4)), который в общем случае - скажем, для неоднородных схем может не иметь места: предел в левой части может не существовать даже для «хороших» управлений или может равняться не числу, а случайной величине. Заглядывая вперед, заметим, что последний аргумент будет важным, если мы рассматриваем не марковскую, а более общую схему (что будет проделано в § 2): в этом случае даже для однородных схем предел в законе больших чисел может оказаться случайным для «хороших» стратегий и достаточно естественных структур зависимости (скажем, для симметрично зависимых с.в.; см., например, [15], [43]).

Во-вторых, определение (1.3) более предпочтительно, чем (1.5), даже если условие (1.4), или аналогичное ему, выполнено. Действительно, пусть для управления $u^{*}$ величина $\lim _{n \rightarrow \infty} n^{-1} J_{x n}\left(u^{*}\right)$, детерминированная или случайная, существует. Тогда из (1.3) следует неравенство

$$
\liminf _{n \rightarrow \infty} n^{-1} J_{x n}(u) \geqslant \lim _{n \rightarrow \infty} n^{-1} J_{x n}\left(u^{*}\right)
$$

- более строгое и более желательное, если это возможно, чем (1.5), так как мы имеем дело не с limsup, a c liminf.

Ниже мы будем следовать определению 1 ввиду того, что, как будет показано, управления со свойством (1.3) существуют при достаточно слабых условиях.

1.2.2. Первое утверждение. Понятие стратегии не является необходимым для дальнейшего изложения, но позволит упростить формулировки. Стратегия - это (конечная или бесконечная) последовательность $\psi=\left(\psi_{1}, \psi_{2}, \ldots\right)$, где измеримая функция $\psi_{t}: \mathbf{R}^{l(t-1)} \rightarrow \mathbf{R}^{m}$ определяет управление в момент $t$; точнее, полагаем $u_{t}=\psi_{t}\left(x^{t-1}\right)$ для $t \geqslant 1$. Если $\psi_{t}\left(x^{t-1}\right)=\varphi_{t}\left(x_{t-1}\right)$ для любого $t$, где $\varphi_{t}: \mathbf{R}^{l} \rightarrow \mathbf{R}^{m}-$ некоторая измеримая функция, то стратегия является марковской. 
Обозначим $U_{\psi x t}, X_{\psi x t}$ реализации управления и процесса соответственно в момент $t$, порожденные стратегией $\psi$ при начальном состоянии $x$. Точнее,

$$
\begin{aligned}
& X_{\psi x 0}=x, \quad X_{\psi x t}=h\left(\xi_{t}, X_{\psi x(t-1)}, U_{\psi x t}\right) \quad \text { при } \quad t=1,2, \ldots, \\
& U_{\psi x 1}=\psi_{1}(x), \quad U_{\psi x t}=\psi_{t}\left(X_{\psi x}^{t-1}\right) \quad \text { при } \quad t=2,3, \ldots .
\end{aligned}
$$

Положим $X_{\psi x}^{t}=\left(X_{\psi x 0}, \ldots, X_{\psi x t}\right)$ и $U_{\psi x}^{n}=\left(U_{\psi x 1}, \ldots, U_{\psi x n}\right)$. Стратегия $\psi$ называется допустимой, если $\left(U_{\psi x 1}, U_{\psi x 2}, \ldots\right) \in \mathscr{U}$ для всех $x$. Процесс, порожденный стратегией, называется траекторией. В обозначениях траекторий мы будем иногда опускать индекс $\psi$ и писать при этом $U_{x t}, X_{x t}$.

Если стратегия определена только до момента $n$ и элементы этой стратегии, возможно, зависят от $n$, то, чтобы это подчеркнуть, мы иногда будем писать $\psi_{t n},{ }_{n} X_{x t},{ }_{n} U_{x t}$. Такая стратегия называется допустимой, если $U_{\psi x}^{n}$ удовлетворяет ограничениям на последовательности из множества $\mathscr{U}$.

Для фиксированного $n$ стратегия $\widehat{\varphi}^{n}$ называется оптимальной в среднем (average optimal), если для каждого начального состояния $x$ управление ${ }_{n} U_{\widehat{\varphi} x t}$, порожденное этой стратегией, оптимально в среднем на интервале $\{0, n\}$. Для краткости будем обозначать такое управление ${ }_{n} \widehat{U}_{x}=\left({ }_{n} \widehat{U}_{x 1}, \ldots,{ }_{n} \widehat{U}_{x n}\right)$, а соответствующую траекторию ${ }_{n} \widehat{X}_{x}=$ $\left({ }_{n} \widehat{X}_{x 1}, \ldots,{ }_{n} \widehat{X}_{x n}\right)$.

В дальнейшем на протяжении всей статьи мы предполагаем, что оптимальная в среднем стратегия существует для любого $n$.

Утверждение 1. Пусть $q(\cdot, \cdot)$ ограничена и существует константа $C$ со следуюшим свойством: для любого $n$ и произвольной парьг (начальных) состояний $s$ u $s^{\prime}$ существуют допустимое управление $z=z^{n}$ и с.в. $N$ (возможно, зависящая от $n, s$ u $s^{\prime}$ ) такие, что

$$
\begin{gathered}
\mathbf{E} N \leqslant C, \\
N<t \leqslant n \Longrightarrow q\left(x_{s t}, z_{t}\right)=q\left({ }_{n} \widehat{X}_{s^{\prime} t},{ }_{n} \widehat{U}_{s^{\prime} t}\right),
\end{gathered}
$$

где $x_{\text {st }}$ - проиесс, порожденный управлением $z$.

Пусть также для некоторого начального состояния $x$ существует управление $\widetilde{U}_{x}=\left\{\widetilde{U}_{x t}\right\}_{t=1}^{\infty} \in \mathscr{U}$ такое, что

$$
\frac{1}{n}\left(J_{x n}\left(\widetilde{U}_{x}\right)-J_{x n}\left({ }_{n} \widehat{U}_{x}\right)\right) \longrightarrow 0 \quad n . H ., \quad n \rightarrow \infty .
$$

Тогда управление $\tilde{U}_{x}$ асимптотически оптимально почти наверное для данного $x$. 
Доказательство будет приведено в разделе 3.1 .

Перейдем к обсуждению. Первые три замечания ниже касаются применений утверждения 1 и условий технического характера.

3 а м е ч а н и я. 1. Прежде всего следует подчеркнуть, что условие допустимости (т.е. принадлежности классу $\mathscr{U}$ ) управления $z$ чисто формально. Покажем, как при применении утверждения 1 к конкретным задачам это условие можно обойти. В дальнейшем это будет проиллюстрировано примерами 1,2 и 4, где в качестве $\mathscr{U}$ рассматривается класс управлений, порожденных стратегиями, а построенные там $z$ не принадлежат этому классу. Действительно, пусть $\mathscr{U}_{1}-$ некоторый класс управлений, включающий в себя $\mathscr{U}$ и такой, что управления, оптимальные в среднем в классах сужений управлений из $\mathscr{U}_{1}$ на всех конечных интервалах, принадлежат соответствующим классам сужений управлений из $\mathscr{U}$. Предположим также, что управление $\widetilde{U}_{x}$ со свойством (1.9) принадлежит $\mathscr{U}$. Это верно, в частности, если $\mathscr{U}_{1}$ - наиболее широкий класс всевозможных управлений, а $\mathscr{U}$ - класс управлений, порожденных стратегиями. Тогда если в классе $\mathscr{U}_{1}$ сушествует управление $z$ со свойствами, указанными в утверждении 1 , применим это утверждение, рассматривая в качестве класса допустимых управлений $\mathscr{U}_{1}$. В результате получим, что в этом классе управление $\widetilde{U}_{x}$ является асимптотически оптимальным почти наверное, а следовательно, оно является таковым и в более узком классе $\mathscr{U}$.

2. Условие ограниченности $q$ не является необходимым: в $\S 2$ оно будет заменено условием на моменты.

3. Как легко видеть, условие (1.9) мы накладываем только потому, что $\left\{{ }_{n} \widehat{U}_{x}\right\}$ представляет собой схему серий, т.е. не принадлежит $\mathscr{U}:$ в противном случае (1.9) было бы излишним и мы говорили бы об оптимальности п.н. самого ${ }_{n} \widehat{U}_{x}$. Хотя в наиболее общем случае, вероятно, достаточно сложно установить условия, при которых управление $\widetilde{U}_{x}$ со свойством (1.9) существует, результаты для некоторых моделей показывают, что эти условия не обременительны. В [27], [12], [38] и многих других статьях управление с упомянутым свойством - это управление, минимизирующее верхний предел среднего по времени значения математического ожидания целевого функционала (the expected value of the long run average cost).

Вернемся теперь к основным условиям (1.7) и (1.8). Формально для выполнения (1.8) можно положить, скажем, $N=n+1$, так как в этом случае множество значений $t$ в левой части (1.8) пусто и, следовательно, (1.8) выполнено автоматически. Но такое $N$ не будет удовлетворять (1.7), так что $N$ не должно зависеть от $n$ «слишком сильно». Можно рассматривать $N$ как время слияния траекторий - время, за которое процесс, стартуя из фиксированного начального состояния $s$, может - при правильно выбранном управлении - достичь траекто- 
рии, начинаюшейся из другого начального состояния $s^{\prime}$ и соответствующей оптимальной в среднем стратегии. Условие состоит в том, чтобы математическое ожидание времени слияния было ограниченным равномерно по $s, s^{\prime} u n$.

Сушественным моментом в условии является равномерность по $s$ и $s^{\prime}$, в то время как $n$ здесь включено только в силу того, что траектория ${ }_{n} \widehat{X}_{s^{\prime} t}$ может зависеть от $n$. С другой стороны, если существует управление $\widetilde{U}_{x}$, удовлетворяющее (1.9), то, грубо говоря, достаточно думать только о времени возможного перехода на траекторию $\widetilde{X}_{x t}$.

Для иллюстрации напомним кратко очень простой пример из [2]; более подробно см. указанную статью. Пусть $x_{t}$ принимает значения $-1,0,1$. Пусть также состояния \pm 1 являются поглощаюшими для любой стратегии, и предположим, что если процесс находится в состоянии 0, то допустимы два управления: при первом процесс остается в 0 с вероятностью 1, в то время как при втором управлении процесс переходит в -1 или в 1 с вероятностями $\frac{1}{2}$. Пусть начальное состояние $x=0$ и $q\left(x_{t}, a_{t}\right)=x_{t}$. Как легко видеть, в этом случае любое управление оптимально в среднем, но не существует управления, оптимального почти наверное.

То же верно и в общем случае: чтобы оптимальное п.н. управление существовало, состояния должны быть в известном смысле сообщающимися. Конечно, в общем случае мы можем потребовать не обязательно точного слияния двух указанных выше траекторий, а только их сближения (см. §2), но некоторое свойство связности должно иметь место. Ввиду этого условия оптимальности п.н., предложенные в данной работе, близки, в определенном смысле, к минимальным.

Перейдем к конкретным примерам. Первый касается классической простой задачи и служит скорее иллюстрацией того, как приведенные выше условия могут быть проверены и почему проверка может оказаться простой и не требовать поиска конкретных управлений, скажем, управлений, оптимальных в среднем.

$\Pi$ р и м е $\mathrm{p} 1$. Обозначим $p_{m}\left(\psi ; n ; s, s^{\prime}\right)$ вероятность того, что не более чем за $m$ шагов следующие две траектории пересекутся: траектория, исходящая из состояния $s$ и порожденная оптимальной в среднем стратегией на интервале $\{0, n\}$, и траектория, исходящая из $s^{\prime}$ и порожденная стратегией $\psi$. Ясно, что $p_{m}$ может обращаться в нуль, при этом являясь неубывающей функцией $m$ для каждой фиксированной $\psi$. Предположим, что для некоторого целого $m$ существует марковская стратегия $\nu=\left(\nu_{1}, \ldots, \nu_{m}\right)$ такая, что для некоторого $q \in(0,1)$

$$
p_{m}\left(\nu ; n ; s, s^{\prime}\right) \geqslant q \text { равномерно по } s, s^{\prime} \text { и } n \text {. }
$$

По крайней мере для конечной цепи условие (1.10) является достаточно слабым. Предположим, например, что класс допустимых страте- 
гий содержит марковскую стратегию $\widetilde{\varphi}$, при которой процесс переходит из любого состояния в другое с вероятностью, большей фиксированного положительного числа. Скажем, при этой стратегии система переходит из одного произвольного состояния в другое с равными вероятностями. Тогда (1.10) выполнено для $\nu=\widetilde{\varphi}$ и $m=1$.

Ясно, что условие утверждения 1 выполнено в случае (1.10).

Действительно, определим управление $z$ из (1.8) следующим образом. Для $t \leqslant m$ положим $z_{t}=U_{\nu s t}$ до момента слияния траекторий, а после этого момента будем управлять процессом в соответствии с оптимальной в среднем стратегией. Если слияние не произошло на интервале до момента $m$, снова начинаем применять стратегию $\nu$ (другими словами, возобновляем управление в соответствии с $\nu$ ), т.е. в этом случае $z_{m+t}=U_{\nu x_{m} t}, t \leqslant m$, и т.д. Заметим, что таким образом мы определили управление, но не стратегию, т.е. управление $z$ может быть таким, что не существует порождающей его стратегии. Пусть $N-$ время слияния траекторий. Для $k$ таких, что $m \leqslant k<2 m$, имеем $\mathbf{P}\{N>k\} \leqslant \mathbf{P}\{N>m\} \leqslant 1-q$. Для $k$ таких, что $l m \leqslant k<(l+1) m$, имеем

$$
\begin{aligned}
\mathbf{P}\{N>k\} \leqslant \mathbf{P}\{N>l m\} & =\mathbf{P}\{N>\operatorname{lm} \mid N>(l-1) m\} \mathbf{P}\{N>(l-1) m\} \\
& \leqslant(1-q) \mathbf{P}\{N>(l-1) m\} .
\end{aligned}
$$

По индукции $\mathbf{P}\{N>k\} \leqslant(1-q)^{[k / m]}$, откуда следует неравенство $\mathbf{E} N \leqslant m / q$.

Следующий пример относится к случаю несчетного множества состояний.

П р и м е р 2. Для простоты рассмотрим сначала управляемую систему вида

$$
x_{t}=h\left(x_{t-1}+u_{t}, \xi_{t}\right)
$$

где $x$ и $u$ теперь имеют одинаковую размерность $(l=m)$, а область значений $h-$ несчетное множество, например, вся числовая прямая. Функция $h$ может быть при этом нелинейной - в случае $l=1$ можно рассмотреть, скажем, $x_{t}=\left(x_{t-1}+u_{t}\right)^{3}+\xi_{t}$, или $x_{t}=\left(x_{t-1}+u_{t}\right)^{3} \xi_{t}$, или $x_{t}=\operatorname{sign}\left(x_{t-1}+u_{t}\right)\left|x_{t-1}+u_{t}\right|^{\xi_{t}}$ и т.д.

В этом случае переход на оптимальную траекторию может быть осушествлен за один шаг: $N=1$. Действительно, чтобы получить

$$
h\left(s+u_{1}, \xi_{1}\right)=h\left(s^{\prime}+\widehat{u}_{1}, \xi_{1}\right),
$$

где $\widehat{u}_{1}=\widehat{u}^{1}\left(s^{\prime}\right)$ соответствует стратегии, оптимальной в среднем, достаточно положить

$$
u_{1}=-s+s^{\prime}+\widehat{u}_{1} .
$$

Хотя для нас это неважно, в этом случае управление $u$ порождается стратегией (зависящей от $s^{\prime}$ ). 
Конечно, (1.11) может быть существенно обобщено. Например, если

$$
x_{t}=h\left(A x_{t-1}+B u_{t}, \xi_{t}\right)
$$

где $A$ и $B$ - невырожденные неслучайные матрицы, или (в одномерном случае) $x_{t}=h\left(q_{1}\left(x_{t-1}\right)+q_{2}\left(u_{t}\right), \xi_{t}\right)$, где $q_{1}$ и $q_{2}$ - взаимно однозначные функции (например, $x_{t}=h\left(x_{t-1}^{3}+u_{t}^{3}, \xi_{t}\right)$ ), вновь очевидным образом существует «переходная» стратегия, для которой $N=1$.

Аддитивность относительно $x_{t-1}$ и $u_{t}$ также не является необходимой. Например, в одномерном случае, чтобы получить то же свойство, достаточно рассмотреть любую систему $x_{t}=h\left(x_{t-1}, u_{t}, \xi_{t}\right)$, где для функции $h$ существует функция $u\left(s, s^{\prime}, \widehat{u}\right)$ (где $\widehat{u}$ - число) такая, что для любого $\xi$

$$
h\left(s, u\left(s, s^{\prime}, \widehat{u}\right), \xi\right)=h\left(s^{\prime}, \widehat{u}, \xi\right) .
$$

В качестве «существенно нелинейного» примера можно рассмотреть

$$
h(s, u, \xi)=\xi(1+|s|)^{u|\xi|} .
$$

(Мультипликативность по $\xi$ не является необходимой; мы это предполагали для красоты примера: если с.в. $\xi$ симметричны, процесс также будет симметричным.) В этом случае $u\left(s, s^{\prime}, \widehat{u}\right)=\widehat{u} \ln \left(1+\left|s^{\prime}\right|\right) / \ln (1+|s|)$.

Теперь рассмотрим примеры, в которых слияние траекторий возможно, но не за один шаг. Сначала рассмотрим случай линейной системы.

П р и м е р 3. Рассмотрим управляемую линейную систему

$$
x_{t}=A x_{t-1}+B u_{t}+\xi_{t} \text {, }
$$

где $\xi_{t}, t=1,2, \ldots$, суть $l$-мерные случайные векторы, случайный вектор $u_{t}, t=1,2, \ldots$, представляет собой управление в момент $t$, а $A$ и $B-$ матрицы соответствующих размеров.

Следует заметить, что, хотя система линейна, пример не связан с линейно-квадратическим регулятором, так как Функиия иень $q-$ - роизвольная ограниченная функция. Условие ограниченности будет ослаблено в $\S 2$.

Если $A$ и $B$ - невырожденные квадратные матрицы, слияние возможно за один шаг, как показано выше. Рассмотрим здесь общий случай. Зафиксируем $n$ и положим

$$
y_{t}={ }_{n} \widehat{X}_{s^{\prime} t}-X_{\varphi s t}, \quad V_{t}={ }_{n} \widehat{U}_{s^{\prime} t}-U_{\varphi s t}, \quad t=1, \ldots, n,
$$

где $\varphi$ обозначает стратегию. Ясно, что

$$
y_{t}=A y_{t-1}+B V_{t}
$$

при $y_{0}=s^{\prime}-s$. 
Известно, что если пара $(A, B)$ является так называемой управляемой парой, то для любого начального состояния существует (неслучайное) управление $V$, которое переводит систему (1.13) из этого состояния в нулевое за $l$ шагов (см., например, $[26])$. Таким образом, существует $V$, переводящее $y_{t}$ из состояния $y_{0}=s^{\prime}-s$ в нулевое состояние.

Точнее, нужно положить $V_{j}=-B^{\prime}\left(A^{\prime}\right)^{l-j-1} W_{l}^{-1} A^{l}\left(s^{\prime}-s\right)$ для $0 \leqslant j \leqslant l-1$ и $V_{j}=0$ для $l \leqslant j \leqslant n$, где $W_{l}=P P^{\prime}$, а $P=$ $\left(B, A B, A^{2} B, \ldots, A^{l-1} B\right)$ - так называемая матрица управляемости; см., например, [26].

При выбранном таким образом $V$ мы выбираем $\varphi$ в соответствии с (1.12), и в этом случае (1.8) выполнено при $N=l$.

Следует заметить, что управление, построенное в последнем примере, порождается стратегией. В общем - нелинейном - случае в ситуации, когда слияние возможно, но не за один шаг, нужное управление может быть построено, но оно не обязательно будет порождаться стратегией.

П р и м е р 4. Рассмотрим процесс вида

$$
x_{t}=U_{t} x_{t-1}+\xi_{t},
$$

где $\xi_{t}, t=1,2, \ldots$, - независимые $l$-мерные случайные векторы, но управлением в момент $t$ является случайная квадратная матрица $U_{t}$, $t=1,2, \ldots$. Пусть $\mathbf{P}\left\{\xi_{t}=0\right\}=p$ для некоторого $p, 0 \leqslant p<1$, и для всех $t$. Если начальное состояние $s$ - некоторый ненулевой вектор, то легко видеть, что требуемое слияние траекторий может быть осуществлено за один шаг. Пусть теперь $s-$ нулевой вектор. Тогда очевидно, что за один шаг обеспечить слияние траекторий невозможно, поэтому построим управление $z=\left(Z_{1}, Z_{2}, \ldots\right)$ следующим образом. Положим $Z_{1}$ нулевой (или любой постоянной) матрицей. В качестве $Z_{2}$ возьмем любую из матриц, удовлетворяющих условию $Z_{2} \xi_{1}={ }_{n} \widehat{X}_{s^{\prime} 2}-\xi_{2}=$ ${ }_{n} \widehat{U}_{s^{\prime} 2} \cdot{ }_{n} \widehat{X}_{s^{\prime} 1}$ (а в дальнейшем управляем в соответствии с оптимальной стратегией) в том случае, если $\xi_{1}$ не является нулевым вектором. В противном случае полагаем $Z_{2}$ нулевой матрицей, а $Z_{3}$ определяем из условия $Z_{3} \xi_{2}={ }_{n} \widehat{X}_{s^{\prime} 3}-\xi_{3}={ }_{n} \widehat{U}_{s^{\prime} 3} \cdot{ }_{n} \widehat{X}_{s^{\prime} 2}$ (и вновь в дальнейшем управляем в соответствии с оптимальной стратегией), если только $\xi_{2}$ не является нулевым вектором, - в противном случае полагаем $Z_{3}$ нулевой матрицей и т.д. Таким образом, слияние траекторий осушествится за два шага для случая, когда $\xi_{1} \neq 0$, т.е. с вероятностью $1-p$, за три шага в случае $\xi_{1}=0, \xi_{2} \neq 0$, т.е. с вероятностью $p(1-p)$ и т.д., - за $k$ шагов с вероятностью $p^{k-2}(1-p), k \leqslant n$. Тогда $\mathbf{E} N \leqslant \sum_{k=2}^{\infty} k p^{k-2}(1-p)=1+(1-p)^{-1}$.

Имеет смысл подчеркнуть, что приведенные примеры служат только иллюстрациями того, как можно применять указанные условия к конкретным задачам. Ясно, что примеры могут быть значительно сложнее. Заметим также, что на самом деле полное совпадение значе- 
ний функций на траекториях не нужно - достаточно сближения этих значений, что в дальнейшем отражено в обшей теореме.

1.3. Оптимальность по вероятности. Обратимся к более слабому определению оптимальности, которое требует и более слабых условий, чем (1.7).

О п р е д е л е н и е 2. Для данного начального состояния $x$ управление $\widetilde{u} \in \mathscr{U}$ называется асимптотически оптимальным по вероятности, если для любого $\varepsilon>0$ и любого управления $u \in \mathscr{U}$

$$
\mathbf{P}\left\{n^{-1}\left[J_{x n}(\widetilde{u})\right]-J_{x n}(u)>\varepsilon\right\} \rightarrow 0, \quad n \rightarrow \infty .
$$

Утверждение 2. Пусть условия на стратегию $\varphi^{n}$ и с.в. $N$ из утверждения 1 выполнень с заменой (1.7) на

$$
\mathbf{P}\{N \geqslant k\}=o\left(k^{-1 / 2}\right) \quad \text { при } \quad k \rightarrow \infty \quad \text { равномерно по } s, s^{\prime} u n .
$$

Пусть также для данного $x$ существует стратегия $\tilde{U}_{x} \in \mathscr{U}$ такая, что для любого $\varepsilon>0$

$$
\mathbf{P}\left\{\frac{1}{n}\left[J_{x n}\left(\widetilde{U}_{x}\right)-J_{x n}\left({ }_{n} \widehat{U}_{x}\right)\right]>\varepsilon\right\} \longrightarrow 0, \quad n \rightarrow \infty .
$$

Тогда $\widetilde{U}_{x}$ асимптотически оптимально по вероятности для данного $x$.

Таким образом, для оптимальности по вероятности не требуется, чтобы математическое ожидание времени перехода было конечным: это время может быть «дольше», и из (1.16) следует, что условие $\mathbf{E} \sqrt{N} \leqslant a$, где $a$ - константа, будет достаточным.

Следует заметить также, что при рассмотрении оптимальности по вероятности мы имеем дело не столько с асимптотическим поведением процесса на бесконечности, сколько с вероятностями определенных множеств для фиксированных (больших) горизонтов планирования. Таким образом, в этом случае, вероятно, будет естественным рассматривать не только бесконечные последовательности $u=\left(u_{1}, u_{2}, \ldots\right)$, но и семейства управлений $\left\{u^{n}\right\}=\left\{u_{1 n}, \ldots, u_{n n}\right\}$, т.е. работать со схемой серий. Это будет проделано в следующем параграфе.

В заключение заметим, что при анализе доказательств в 3 , так же как и во многих упомянутых работах, можно увидеть, что для того, чтобы получить аналогичные результаты, достаточно делить выражение $J_{x n}(u)-J_{x n}\left(u^{*}\right)$ не на $n$, а на некоторую функцию $r(n)=o(n)$. $\mathrm{B}$ частности, это связано с тем фактом, что мы исходим из закона больших чисел для мартингалов с конечными дисперсиями. Задача в указанной постановке исследовалась в [16].

До сих пор в общем случае не ясен наилучший порядок функции $r$. Понятно, что можно использовать $r(n)$ такую, что $r(n) / \sqrt{n} \rightarrow \infty$ (см., 
например, [29], где исследовались марковские цепи), но было показано, что, по крайней мере в некоторых частных случаях, этот результат может быть улучшен. Это касается, например, линейной модели с квадратичным функционалом, исследованной с этой точки зрения (для случая непрерывного времени) в [27] (где рассматривалась так называемая «overtaking optimality») и в [39], [7]. В [39] было показано, что для данной модели достаточно делить на любую $r(n) \rightarrow \infty$ для оптимальности по вероятности, а в [7] - что для оптимальности п.н. нужно делить на $r(n)$ такую, что $r(n) / \ln n \rightarrow \infty$, причем этот порядок является наилучшим; подробнее см. упомянутые работы. Конкретная форма целевого функционала и линейность управления в указанной модели являются существенными. При этом остается неясным, как этот результат может быть распространен на общий случай.

\section{$\S 2$. Общая постановка}

Сформулируем обобщение приведенной выше схемы следующим образом.

1. Будем рассматривать более общие, в частности, неоднородные процессы.

2. Условие (1.8) требует полного слияния двух определенных траекторий. Ниже будем предполагать, что эти траектории лишь приближаются друг к другу.

3. Вместо ограниченности функции $q$ будем требовать ниже в теореме 1 ограниченность моментов некоторых характеристик.

4. Класс допустимых управлений ниже может включать не только бесконечные последовательности управлений, но и схемы серий управлений.

Общая схема стохастической динамической оптимизации в дискретном времени может быть представлена следующим образом.

Пусть $\left\{\Omega, \mathscr{F}, \mathscr{F}^{t}, \mathbf{P}\right\}$ - вероятностное пространство с фильтрацией $\left\{\mathscr{F}^{t} \subset \mathscr{F}\right\}_{t=0}^{\infty}$ и $\left\{\left(A_{t}, \mathscr{A}_{t}\right)\right\}_{t=1}^{\infty}$ - последовательность измеримых пространств произвольной природы.

Фильтрация $\left\{\mathscr{F}^{t}\right\}$ соответствует рассматриваемому процессу: нет необходимости вводить этот процесс в явном виде. Сигма-алгебра $\mathscr{F}^{0}$ «соответствует возможным начальным состояниям» процесса. Если $\mathscr{F}^{0}$ тривиальна, мы интерпретируем это как то, что начальное состояние фиксировано.

Пространство $\left(A_{t}, \mathscr{A}_{t}\right)$ интерпретируется как пространство решений, принимаемых в момент $t$.

Положим $A^{t}=A_{1} \times \cdots \times A_{t}, \mathscr{A}^{t}=\mathscr{A}_{1} \times \cdots \times \mathscr{A}_{t}$ (прямое произведение), $a^{t}=\left(a_{1}, \ldots, a_{t}\right)$ при $a_{j} \in A_{j}$. 
Для $n \geqslant 1$ определим теперь последовательность аддитивных целевых функционалов

$$
J_{n}\left(\omega, a^{n}\right)=\sum_{t=1}^{n} g_{t}\left(\omega, a^{t}\right),
$$

где функция $g_{t}\left(\omega, a^{t}\right)$ является $\left(\mathscr{F}^{t} \times \mathscr{A}^{t}\right)$-измеримой. Представление (2.1), конечно, позволяет включать в рассмотрение управляемые процессы. Пусть, например, верно (1.1), тогда для некоторой измеримой функции $h^{\prime}: \mathbf{R}^{t} \times \mathbf{R}^{m t} \rightarrow \mathbf{R}^{l}$ можно записать $x_{t}=h^{\prime}\left(\xi^{t}, a^{t}\right)$, следовательно, $g_{t}\left(\omega, a^{t}\right)=q\left(h^{\prime}\left(\xi^{t}, a^{t}\right), a_{t}\right)$, где $q-$ функция из представления функционала (1.2). При этом ясно, что представление (2.1) включает возможность рассмотрения и неоднородных процессов, когда функция $h$ явно зависит от времени.

Заметим, что в общем случае можно наделить $g_{t}$ вторым индексом $n$, допуская, что $g_{t}$ может зависеть от горизонта планирования. Мы опускаем его здесь, чтобы избежать громоздкости формул, хотя в определенном смысле запись $g_{t n}$ будет более уместна, чем $g_{t}$. В этом случае можно считать, что нормирование суммы (скажем, делением на $n$ ) уже проделано. Например, вместо $n^{-1} \sum_{t=1}^{n} g_{t}\left(\omega, a^{t}\right)$ можно писать $\sum_{t=1}^{n} g_{t n}\left(\omega, a^{t}\right)$, полагая $g_{t n}\left(\omega, a^{t}\right)=g_{t}\left(\omega, a^{t}\right) / n$. Тем не менее в данной работе мы будем иметь дело с традиционным видом записи, чтобы сделать порядок некоторых асимптотических соотношений более явным.

Будем писать иногда $g_{t}\left(\omega, a^{n}\right)$ вместо $g_{t}\left(\omega, a^{t}\right)$, имея при этом в виду, что $g_{t}$ зависит только от первых $t$ координат вектора $a^{n}$.

Для фиксированного $n$ допустимое управление представляет собой вектор $u^{n}=\left(u_{1 n}, \ldots, u_{n n}\right)$, где $u_{t n}: \Omega \rightarrow \mathscr{A}_{t}$ является $\mathscr{F}^{t-1}$-измеримым, так что управление может зависеть от горизонта планирования. Иногда, если это не приводит к неправильному пониманию, мы будем опускать индекс $n$. То же касается стратегий, используемых ниже.

Пусть $\mathscr{U}_{n}$ - класс допустимых управлений для фиксированного $n$, $\mathscr{U}=\left\{\mathscr{U}_{n}\right\}$ и $u=\left\{u^{n}\right\}$, где $u^{n} \in \mathscr{U}_{n}$. В общем случае $u$ представляет собой схему серий. Чтобы свести эту схему к традиционной постановке, мы должны предположить, что для каждого $u=\left\{u^{n}\right\} \in \mathscr{U}$ верно равенство $u_{t n}=u_{t t}$ при $n>t$. Как и в предыдущем параграфе, читатель при желании может иметь в виду традиционную схему, игнорируя второй индекс $n$.

Используя, как и выше, греческие буквы для стратегий, определим для фиксированного $n$ стратегию как вектор $\varphi^{n}=\left\{\varphi_{1}, \ldots, \varphi_{n}\right\}$, где $\varphi_{1}: \Omega \rightarrow \mathscr{A}_{1}$ есть $\mathscr{F}^{0}$-измеримое отображение и $\varphi_{t}: \Omega \times A^{t-1} \rightarrow A_{t}$ является $\left(\mathscr{F}^{t-1} \times \mathscr{A}^{t-1}\right)$-измеримым для $t=2,3, \ldots$

Проиллюстрируем приведенную схему на примере марковской цепи, рассмотренной в разделе 1.2. Пусть с.в. $\xi_{t}$ из (1.1) определены на пространстве $\{\Omega, \mathscr{F}, \mathbf{P}\}, \mathscr{F}^{0}$ тривиальна и $\mathscr{F}^{t}=\sigma\left(\xi^{t}\right)$. В силу (1.1) суще- 
ствует $\left(\mathscr{F}^{t} \times \mathscr{A}^{t}\right)$-измеримая функция $g_{t}\left(\omega, a^{t}\right)=q\left(x_{t}, a_{t}\right)$. Для любой стратегии $\psi_{t}\left(x^{t-1}\right)$ из раздела 1.2 существует $\left(\mathscr{F}^{t-1} \times \mathscr{A}^{t-1}\right)$-измеримая функция $\varphi\left(\omega, a^{t-1}\right)=\psi_{t}\left(x^{t-1}\right)$. (Обе функции $g$ и $h$ могут зависеть от фиксированного начального состояния $x$.) Таким образом, мы оказываемся в рамках описанной выше схемы.

Описанная схема позволяет избежать некоторых излишних рассмотрений и делает более простыми доказательства. Конечно, это не означает, что знание конкретной структуры процесса (скажем, представления (1.1) и (1.2)) не может быть полезно для других целей. Заметим также, что дополнительная информация о процессе и о целевом функционале может быть описана в терминах $\sigma$-алгебр, относительно которых функции $g_{t}$ измеримы. В частных моделях эти $\sigma$-алгебры, как правило, проше, чем $\mathscr{F}^{t} \times \mathscr{A}^{t}$ (см., например, [40]).

Кроме того, для каждой стратегии $\varphi^{n}$ определим набор операторов ${ }_{t} \Phi^{n}, t=1, \ldots, n$, переводящих пространство управлений в себя, таких, что управление ${ }_{t} \Phi^{n} u^{n}$ совпадает с $u^{n}$ до момента $t$, а начиная с этого момента ${ }_{t} \Phi^{n} u^{n}$ управляет процессом в соответствии со стратегией $\varphi^{n}$. Точнее, для $\widetilde{u}^{n}=\left(\widetilde{u}_{1}, \ldots, \widetilde{u}_{n}\right)={ }_{t} \Phi^{n} u^{n}$

$$
\widetilde{u}_{j}=\left\{\begin{array}{lll}
u_{j}, & \text { если } j<t ; \\
\varphi_{j}\left(\omega, \widetilde{u}_{1}(\omega), \ldots, \widetilde{u}_{j-1}(\omega)\right), & \text { если } j \geqslant t .
\end{array}\right.
$$

Управление ${ }_{1} \Phi^{n} u^{n}$ - это управление, порожденное стратегией $\varphi^{n} \mathrm{c}$ начального момента, так что оно не зависит от $u^{n}$. Будем считать, что ${ }_{t} \Phi^{n} u^{n}=u^{n}$ при $t>n$.

Положим $\mathbf{E}_{t}(\cdot)=\mathbf{E}\left(\cdot \mid \mathscr{F}^{t-1}\right), \mathbf{E}_{0}=\mathbf{E}$.

В неоднородном случае нам понадобится немного более строгое понятие, чем оптимальность в среднем. А именно, предположим, что существует постоянно оптимальная стратегия $\widehat{\varphi}^{n}$, т.е. стратегия такая, что для всех $u^{n} \in \mathscr{U}_{n}$ и $t=0,1, \ldots, n$

$$
\mathbf{E}_{t} J_{n}\left(\omega,{ }_{t+1} \widehat{\Phi}^{n} u^{n}\right) \geqslant \mathbf{E}_{t} J_{n}\left(\omega,{ }_{t} \widehat{\Phi}^{n} u^{n}\right) \quad \text { п.н., }
$$

где операторы ${ }_{t} \widehat{\Phi}^{n}$ соответствуют $\widehat{\varphi}^{n}$. (О постоянной оптимальности см., например, [25].) Положим $\widehat{u}^{n}={ }_{1} \widehat{\Phi}^{n} u^{n}$ и $\widehat{u}=\left\{\widehat{u}^{n}\right\}$.

Далее введем некоторые условия, которые не обязаны будут выполняться одновременно.

У с л о в и е А. Существуют $p>2$ и константа $A_{p}$ со следующим свойством: для любого $n$, любой пары управлений $u^{n}, \bar{u}^{n} \in \mathscr{U}_{n}$ и всех $t \leqslant n-1$ найдется управление $z^{n} \in \mathscr{U}_{n}$ такое, что

$$
z^{n}=\left(\bar{u}_{1}, \ldots, \bar{u}_{t}, z_{t+1}, \ldots, z_{n}\right)
$$

и

$$
\mathbf{E}\left|\sum_{j=t+1}^{n}\left\{g_{j}\left(\omega, z^{n}\right)-g_{j}\left(\omega,{ }_{t+1} \widehat{\Phi}^{n} u^{n}\right)\right\}\right|^{p} \leqslant A_{p} .
$$


Так как в общем случае левая часть (2.4) имеет порядок $n-t$, неравенство (2.4) может быть выполнено, если значения цены $g_{j}(\cdot, \cdot)$ для двух управлений, $z^{n}$ и $_{t+1} \widehat{\Phi}^{n} u^{n}$, близки друг к другу для больших $j$. При этом условие А означает существование управления $z^{n}$, для которого это верно.

Как альтернативу условию А можно рассмотреть следующее условие.

У с л о в и е В. Существует константа $B$ такая, что для любого $n$, любой пары управлений $u^{n}, \bar{u}^{n} \in \mathscr{U}_{n}$ и всех $t \leqslant n-1$ найдется управление (2.3) такое, что

$$
\mathbf{E}_{t+1}\left|\sum_{j=t+1}^{n}\left\{g_{j}\left(\omega, z^{n}\right)-g_{j}\left(\omega,{ }_{t+1} \widehat{\Phi}^{n} u^{n}\right)\right\}\right| \leqslant B \quad \text { п.н. }
$$

Таким образом, если мы накладываем условие равномерной ограниченности условного математического ожидания, то используем только первый момент, в то время как в случае ограничения на безусловное математическое ожидание используем моменты более высоких порядков.

Для оптимальности по вероятности достаточно накладывать более слабые условия.

У с л о в и е $\mathrm{A}^{\prime}$. Сушествует функция $\gamma(n) \rightarrow 0, n \rightarrow \infty$, со следующим свойством: для всех $n$, всех пар $u^{n}, \bar{u}^{n} \in \mathscr{U}_{n}$ и всех $t \leqslant n-1$ найдется управление $z^{n} \in \mathscr{U}_{n}$ такое, что выполнено (2.3) и

$$
\mathbf{E}\left[\sum_{j=t+1}^{n}\left\{g_{j}\left(\omega, z^{n}\right)-g_{j}\left(\omega, t+1 \widehat{\Phi}^{n} u^{n}\right)\right\}\right]^{2} \leqslant \gamma(n) n .
$$

У с л о в и е $\mathrm{B}^{\prime}$. Условие $\mathrm{A}^{\prime}$ верно с заменой (2.6) на

$$
\mathbf{E}_{t+1}\left|\sum_{j=t+1}^{n}\left\{g_{j}\left(\omega, z^{n}\right)-g_{j}\left(\omega,{ }_{t+1} \widehat{\Phi}^{n} u^{n}\right)\right\}\right| \leqslant \gamma(n) \sqrt{n} .
$$

Теорема 1. (1) Пусть выполнено одно из условий А или В и для всех $u=\left\{u^{n}\right\} \in \mathscr{U}$

$$
\frac{1}{n} \sum_{t=1}^{n}\left\{g_{t}\left(\omega, u^{n}\right)-\mathbf{E}_{t} g_{t}\left(\omega, u^{n}\right)\right\} \longrightarrow 0 \quad n p u \quad n \rightarrow \infty
$$

почти наверное. Тогда последовательность управлений $\widehat{u}$ асимптотически оптимальна почти наверное.

(2) Если выполнено одно из условий $\mathrm{A}^{\prime}$ или $\mathrm{B}^{\prime}$ и для всех $u=$ $\left\{u^{n}\right\} \in \mathscr{U}(2.8)$ вылолнено по вероятности, то последовательность $\widehat{u}$ асимптотически оптимальна по вероятности. 
Следующие замечания касаются достаточных условий справедливости (2.8).

1. Так как слагаемые в (2.8) являются мартингал-разностями относительно $\left\{\mathscr{F}^{t}\right\}$, ясно, что (2.8) выполнено по вероятности, если, скажем, для всех $u$

$$
\frac{1}{n^{2}} \sum_{t=1}^{n} \mathbf{E}\left[g_{t}\left(\omega, u^{n}\right)-\mathbf{E}_{t} g_{t}\left(\omega, u^{n}\right)\right]^{2} \longrightarrow 0 \quad \text { при } \quad n \rightarrow \infty
$$

Это уже вполне слабое условие.

2. Очевидно, (2.9) выполнено, если функции равномерно ограничены, т.е.

$$
\sup _{t, \omega, a^{t}}\left|g_{t}\left(\omega, a^{t}\right)\right|<\infty .
$$

3. Используя лемму Бореля-Кантелли, нетрудно получить (см. также аргументы в (3.9) ниже), что (2.8) выполнено почти наверное, если для некоторого $p \geqslant 2$ и всех $u \in \mathscr{U}$

$$
\sum_{n=1}^{\infty} \frac{1}{n^{p}}\left[\sum_{t=1}^{n}\left\|g_{t}\left(\omega, u^{n}\right)-\mathbf{E}_{t} g_{t}\left(\omega, u^{n}\right)\right\|_{p}^{2}\right]^{p / 2} \cdot<\infty
$$

где $\|\cdot\|_{p}$ обозначает норму в $L_{p}$.

4. Ясно, что (2.11) выполнено, если, например, существуют константы $C>0$ и $p>2$ такие, что для всех управлений $u^{n} \in \mathscr{U}_{n}$

$$
\left\|g_{t}\left(\omega, u^{t}\right)-\mathbf{E}_{t} g_{t}\left(\omega, u^{t}\right)\right\|_{p} \leqslant C .
$$

Очевидно, (2.10) влечет (2.12).

\section{$\S$ 3. Доказательства}

3.1. Доказательства утверждений 1 и 2. Покажем, что эти утверждения следуют из теоремы 1. Мы предположили существование стратегий $\widehat{\varphi}^{n}$, оптимальных в среднем на $\{0, n\}$, т.е. стратегий, порождающих оптимальные в среднем управления для всех начальных состояний. Так как схема однородна, это означает, что $\widehat{\varphi}^{n}$ постоянно оптимальны. Так что мы можем зафиксировать начальное состояние $x$ и рассмотреть проблему в рамках приведенной выше обшей схемы, предполагая, что $\mathscr{F}^{0}$ тривиальна.

В нашем случае $g_{t}\left(\omega, u^{n}\right)=q\left(x_{t}, u_{t}\right)$, где $x_{t}$ - процесс, порожденный $u$. Так как $q$ ограничена, (2.8) в этом случае выполнено автоматически по вероятности и почти наверное (см. также замечания после теоремы 1).

Покажем теперь, что из условия утверждения 1 следует условие В, т.е. (2.5). Так как схема однородна и $\sigma$-алгебра $\mathscr{F}^{0}$ тривиальна, достаточно проверить (2.5) для $t=0$ и заменить условное математическое 
ожидание на безусловное. Пусть $N$ и $\varphi-$ с.в. и стратегия соответственно, определенные в утверждении 1. Тогда в силу (1.8)

$$
\left|\sum_{j=1}^{n}\left\{g_{j}\left(\omega, z^{n}\right)-g_{j}\left(\omega,{ }_{1} \widehat{\Phi}^{n} u^{n}\right)\right\}\right| \leqslant 2 N G,
$$

где $G=\sup _{x, a}|q(x, a)|$. Таким образом, если $\mathbf{E} N \leqslant C$, то (2.5) выполнено при $B=2 G C$.

Рассмотрим теперь оптимальность по вероятности, т.е. утверждение 2. В этом случае достаточно проверить (2.7) для $t=0$ и заменить условное математическое ожидание безусловным. Тогда (2.7) следует из (1.16).

Заметим, что если $N \geqslant n$, то левая часть (1.8) является пустым множеством и, значит, (1.8) выполнено автоматически. Следовательно, (1.8) останется верным, если мы заменим с.в. $N$ ее усечением $N^{(n)}=N$, при $N<n$ и $N^{(n)}<n$ при $N \geqslant n$. Это означает, что в правой части $(3.1)$ можно заменить $N$ на $N^{(n)}$.

В силу (1.16) существует функция $\delta_{0}(k) \rightarrow 0$ при $k \rightarrow \infty$, одна и та же для всех $s, s^{\prime}$ и $n$ и такая, что $\mathbf{P}\{N>k\} \leqslant \delta_{0}(k) / \sqrt{k}$. Следовательно,

$$
\mathbf{E} N^{(n)}=\mathbf{E}\{N ; N \leqslant n\}+n \mathbf{P}\{N>n\} \leqslant \mathbf{E}\{N ; N \leqslant n\}+\delta_{0}(n) \sqrt{n} .
$$

Ясно, что из (1.16) следует, что $\mathbf{E}\{N ; N \leqslant n\} \leqslant \delta_{1}(n) \sqrt{n}$, где функция $\delta_{1}(n) \rightarrow 0$ и определяется только функцией $\delta_{0}(n)$.

Следовательно, в рассматриваемом случае из (1.8) следует (2.7) при $\gamma(n)=2 G\left(\delta_{1}(n)+\delta_{0}(n)\right)$.

3.2. Доказательство теоремы 1. Будем использовать некоторые факты из [2] и [42]. Рассмотрим сначала стратегию $\widehat{\varphi}^{n}$, управление $\widehat{u}^{n}$ и отображение ${ }_{t} \widehat{\Phi}^{n}$, определенные в $\S 2$. Положим $\widehat{u}=\left\{\widehat{u}^{n}\right\} \in \mathscr{U}$ и зафиксируем другую последовательность управлений $u=\left\{u^{n}\right\}$.

Ниже будем опускать индекс $n$ в $u_{t n}, \widehat{u}_{t n}, \widehat{\varphi}_{t n}$ и $_{t} \widehat{\Phi}^{n}$.

Пусть с.в.

$$
Y_{t}=Y_{t n}=\mathbf{E}_{t} J_{n}\left(\omega,{ }_{t+1} \widehat{\Phi} u^{n}\right)-\mathbf{E}_{t} J_{n}\left(\omega,{ }_{t} \widehat{\Phi} u^{n}\right)
$$

Нетрудно увидеть, что

$J_{n}\left(\omega, u^{n}\right)=\sum_{t=0}^{n}\left(\mathbf{E}_{t+1}-\mathbf{E}_{t}\right) J_{n}\left(\omega, t+1, \widehat{\Phi} u^{n}\right)+\sum_{t=1}^{n} Y_{t}+\mathbf{E} J_{n}\left(\omega, \widehat{u}^{n}\right)$.

В частности, если $u=\widehat{u}$, то $Y_{t}=0$ п.н. и

$$
J_{n}\left(\omega, \widehat{u}^{n}\right)=\sum_{t=0}^{n}\left(\mathbf{E}_{t+1}-\mathbf{E}_{t}\right) J_{n}\left(\omega, \widehat{u}^{n}\right)+\mathbf{E} J_{n}\left(\omega, \widehat{u}^{n}\right) .
$$

Вычитая равенство (3.3) из (3.2), получим (см. также [2])

$$
J_{n}\left(\omega, u^{n}\right)-J_{n}\left(\omega, \widehat{u}^{n}\right)=\sum_{t=1}^{n} Y_{t}+\sum_{t=1}^{n} W_{t}
$$


где

$$
\begin{aligned}
& W_{t}=W_{t n}=\sum_{j=t}^{n}\left[Z_{t j}-\mathbf{E}_{t} Z_{t j}\right], \quad Z_{t t}=Z_{t t}(n)=g_{t}\left(\omega, u^{n}\right)-g_{t}\left(\omega, \widehat{u}^{n}\right), \\
& Z_{t j}=Z_{t j}(n)=\mathbf{E}_{t+1}\left\{g_{j}\left(\omega, t+1, \widehat{\Phi} u^{n}\right)-g_{j}\left(\omega, \widehat{u}^{n}\right)\right\} \quad \text { при } \quad j>t .
\end{aligned}
$$

(Следует напомнить здесь, что $g_{j}\left(\omega, u^{n}\right)$ зависит только от первых $j$ координат вектора $u^{n}=\left(u_{1}, \ldots, u_{n}\right)$ и $\mathbf{E}_{t} Z_{t j}=\mathbf{E}_{t+1} Z_{t j}$ при $t \geqslant j$.)

Заметим, что, взяв математическое ожидание в (3.4), мы придем к соотношению

$$
\mathbf{E} J_{n}\left(\omega, u^{n}\right)-\mathbf{E} J_{n}\left(\omega, \widehat{u}^{n}\right)=\sum_{t=1}^{n} \mathbf{E} Y_{t}
$$

которое аналогично формуле для «дефекта стратегии», определенного в [45]. Представление (3.4) включает в рассмотрение также «случайную часть дефекта».

Так как стратегия $\widehat{\varphi}^{n}$ постоянно оптимальна, то $Y_{t} \geqslant 0$ п.н. для всех $t \leqslant n$ (см. (2.2)). Следовательно, осталось доказать, что

$$
\frac{1}{n} \sum_{t=1}^{n} W_{t} \rightarrow 0 \quad \text { при } \quad n \rightarrow \infty
$$

почти наверное или по вероятности, в зависимости от рассматриваемого типа оптимальности.

Пусть

$$
\begin{aligned}
& V_{t}=V_{t n}=\sum_{j=t+1}^{n} \mathbf{E}_{t+1} g_{j}(\omega, t+1 \\
& \left.\widehat{V}_{t} u^{n}\right) \\
& =\widehat{V}_{t n}=\sum_{j=t+1}^{n} \mathbf{E}_{t+1} g_{j}\left(\omega, \widehat{u}^{n}\right), \quad R_{t}=R_{t n}=V_{t n}-\widehat{V}_{t n} .
\end{aligned}
$$

Тогда

$$
W_{t}=Z_{t t}-\mathbf{E}_{t} Z_{t t}+R_{t}-\mathbf{E}_{t} R_{t}
$$

В силу условия (2.8)

$$
\frac{1}{n} \sum_{t=1}^{n}\left[Z_{t t}-\mathbf{E}_{t} Z_{t t}\right] \longrightarrow 0 \quad \text { при } \quad n \rightarrow \infty
$$

по вероятности или п.н. (см. формулировку теоремы 1). Таким образом, достаточно показать, что

$$
\frac{1}{n} \sum_{t=1}^{n-1}\left\{R_{t}-\mathbf{E}_{t} R_{t}\right\} \longrightarrow 0 \quad \text { при } \quad n \rightarrow \infty
$$

по вероятности или п.н. 
Зафиксируем некоторое $p>2$ и положим $U_{t}=R_{t}-\mathbf{E}_{t} R_{t}$. Слагаемые в (3.8) суть мартингал-разности относительно $\left\{\mathscr{F}^{t}\right\}$, и в силу неравенства Буркхольдера существует константа $C_{p}$, зависящая только от $p$, такая, что

$$
\begin{aligned}
\mathbf{E}\left|\sum_{t=1}^{n-1} U_{t}\right|^{p} & \leqslant C_{p} \mathbf{E}\left|\sum_{t=1}^{n-1} U_{t}^{2}\right|^{p / 2}=C_{p}\left\|\sum_{t=1}^{n-1} U_{t}^{2}\right\|_{p / 2}^{p / 2} \leqslant C_{p}\left[\sum_{t=1}^{n-1}\left\|U_{t}^{2}\right\|_{p / 2}\right]^{p / 2} \\
& =C_{p}\left[\sum_{t=1}^{n-1}\left\|U_{t}\right\|_{p}^{2}\right]^{p / 2} \leqslant 2^{p} C_{p}\left[\sum_{t=1}^{n-1}\left\|R_{t}\right\|_{p}^{2}\right]^{p / 2}
\end{aligned}
$$

Используя (3.9), неравенство Маркова и лемму Бореля-Кантелли, легко показать, что сходимость почти наверное в (3.8) имеет место, если для некоторого $p>2$

$$
\sum_{n=1}^{\infty} \frac{1}{n^{p}}\left[\sum_{t=1}^{n-1}\left\|R_{t}\right\|_{p}^{2}\right]^{p / 2}<\infty
$$

Зафиксируем $n$ и $t \leqslant n$ и вернемся к условию А или $\mathrm{B}$, где рассматривается пара произвольных управлений $\left(u^{n}, \bar{u}^{n}\right)$. Положим сначала $\bar{u}=\widehat{u}$. Тогда управление (2.3) имеет вид

$$
z^{n}=\left(\widehat{u}_{1}, \ldots, \widehat{u}_{t}, z_{t+1}, \ldots, z_{n}\right) .
$$

Так как стратегия $\widehat{\varphi}^{n}$ постоянно оптимальна, то

$$
\widehat{V}_{t} \leqslant \mathbf{E}_{t+1} \sum_{j=t+1}^{n} g_{j}\left(\omega, z^{n}\right)=V_{t}+\mathbf{E}_{t+1}\left(\sum_{j=t+1}^{n} g_{j}\left(\omega, z^{n}\right)-\sum_{j=t+1}^{n} g_{j}\left(\omega, t_{t+1} \widehat{\Phi}^{n} u^{n}\right)\right)
$$

и

$$
\widehat{V}_{t}-V_{t} \leqslant\left|\mathbf{E}_{t+1}\left(\sum_{j=t+1}^{n} g_{j}\left(\omega, z^{n}\right)-\sum_{j=t+1}^{n} g_{j}\left(\omega, t+1 \widehat{\Phi}^{n} u^{n}\right)\right)\right|
$$

Рассмотрим теперь в качестве пары $\left(u^{n}, \bar{u}^{n}\right)$ пару $\left(\widehat{u}, t+1 \widehat{\Phi}^{n} u^{n}\right)$. Обозначим управление $(2.3)$ в этом случае $k^{n}$. Ясно, что оно отличается от управления (3.11). По определению оператора ${ }_{t+1} \widehat{\Phi}^{n}$ имеем $\bar{u}_{j}=u_{j}$ для $j \leqslant t$, и управление (2.3) может быть представлено в этом случае в виде

$$
k^{n}=\left\{u_{1}, \ldots, u_{t}, k_{t+1}, \ldots, k_{n}\right\} .
$$

Заметим также, что ${ }_{t+1} \widehat{\Phi}^{n} \widehat{u}^{n}=\widehat{u}^{n}$ для всех $t$. Поэтому в силу постоянной оптимальности стратегии $\widehat{\varphi}^{n}$ имеем

$$
\begin{aligned}
V_{t} \leqslant & \mathbf{E}_{t+1} \sum_{j=t+1}^{n} g_{j}\left(\omega, k^{n}\right)=\mathbf{E}_{t+1} \sum_{j=t+1}^{n} g_{j}(\omega, t+1 \\
& +\mathbf{E}_{t+1}\left(\sum_{j=t+1}^{n} \widehat{u}^{n}\right) \\
& \left.g_{j}\left(\omega, k^{n}\right)-\sum_{j=t+1}^{n} g_{j}\left(\omega, t+1 \widehat{\Phi}^{n} \widehat{u}^{n}\right)\right)
\end{aligned}
$$




$$
\begin{aligned}
& =\mathbf{E}_{t+1} \sum_{j=t+1}^{n} g_{j}\left(\omega, \widehat{u}^{n}\right)+\mathbf{E}_{t+1}\left(\sum_{j=t+1}^{n} g_{j}\left(\omega, k^{n}\right)-\sum_{j=t+1}^{n} g_{j}(\omega, t+1\right. \\
& =\widehat{\Phi}_{t}+\mathbf{E}_{t+1}\left(\sum_{j=t+1}^{n} g_{j}\left(\omega, k^{n}\right)-\sum_{j=t+1}^{n} g_{j}(\omega, t+1\right.
\end{aligned}
$$

и

$$
V_{t}-\widehat{V}_{t} \leqslant\left|\mathbf{E}_{t+1}\left(\sum_{j=t+1}^{n} g_{j}\left(\omega, k^{n}\right)-\sum_{j=t+1}^{n} g_{j}\left(\omega, t+1 \widehat{\Phi}^{n} \widehat{u}^{n}\right)\right)\right| .
$$

Таким образом, если условие А выполнено, то в силу (3.12) и (3.13)

$$
\left\|R_{t}\right\|_{p}=\left\|\widehat{V}_{t}-V_{t}\right\|_{p} \leqslant 2 A_{p}^{1 / p}
$$

и

$$
\sum_{n=1}^{\infty} \frac{1}{n^{p}}\left[\sum_{t=1}^{n-1}\left\|R_{t}\right\|_{p}^{2}\right]^{p / 2} \leqslant 2^{p} A_{p} \sum_{n=1}^{\infty} \frac{1}{n^{p / 2}}<\infty
$$

для $p>2$.

Пусть теперь выполнено условие В. В этом случае из (3.12), (3.13) и (2.5) следует, что $\left|R_{t}\right|=\left|V_{t}-\widehat{V}_{t}\right| \leqslant 2 B$ п.н., и левая часть (3.15) снова конечна для $p>2$. Таким образом, первое утверждение теоремы 1 доказано.

Рассмотрим теперь сходимость в (3.8) по вероятности. Аналогично доказательству неравенства (3.14) можно получить, что при условии $\mathrm{A}^{\prime}$

$$
\mathbf{E}\left|R_{t}\right|^{2} \leqslant 2 \gamma(n) n
$$

Тогда дисперсия левой части (3.8) не превосходит

$$
\frac{1}{n^{2}} \sum_{t=1}^{n} 2 \mathbf{E}\left|R_{t}\right|^{2} \leqslant 4 \gamma(n) \longrightarrow 0 .
$$

В случае выполнения условия $\mathrm{B}^{\prime}$ имеем

$$
\left|R_{t}\right| \leqslant 2 \gamma(n) \sqrt{n} \quad \text { п.н., }
$$

и (3.16) снова верно.

\section{СПИСОК ЛИТЕРАТУРЫ}

1. Аркин В.И., Евстигнеев И. В. Вероятностные модели управления и экономической динамики. М.: Наука, 1979.

2. Asriev A.V., Rotar V.I. On asymptotic optimality in probability and almost surely in dynamic control. - Stochastics Stochastics Rep., 1990, v. 33, № 1-2, p. 1-16.

3. Конюхова (Белкина) T. А. Асимптотически оптимальные по вероятности управления в задаче о линейном регуляторе с переменными параметрами. - Автоматика и телемеханика, 1994, т. 55, № 2, с. 110-120. 
4. Белкина Т. А., Пресман Э. Л. Асимптотически оптимальные по распределению - управления для линейной стохастической системы с квадратичным функционалом. - Автоматика и телемеханика, 1997, т. 58, № 3, с. 106-115.

5. Конюхова (Белкина) Т. А., Ротарь В. И. Управления, асимптотически оптимальные по вероятности и почти наверное, в задаче о линейном регуляторе. - Автоматика и телемеханика, 1992, т. 53, №6, с. 65-78.

6. Белкина Т. А., Ротарь В. И. Об условиях асимптотической оптимальности по вероятности и почти наверное в модели управляемого диффузионного процесса. Автоматика и телемеханика, 1999, т. 60, № 2, с. 45-56.

7. Белкина Т. А., Кабанов Ю. М., Пресман Э. Л. О стохастической оптимальности для линейно-квадратического регулятора. - Теория вероятн. и ее примен., 2003, т. 48 , в. 4 , c. $661-675$.

8. Bhattacharia R. N. Asymptotic behavior of several-dimensional diffusions. - Stochastic Nonlinear Systems in Physics, Chemistry, and Biology. Ed. by L. Arnold and R. Lefever. Berlin-New York: Springer-Verlag, 1981, p. 86-99.

9. Borkar V.S. Optimal Control of Diffusion Processes. New York: Longman, 1989, $196 \mathrm{p}$.

10. Borkar V.S. A convex analytic approach to Markov decision processes. - Probab. Theory Related Fields, 1988, v. 78, № 4, p. 583-602.

11. Borkar V.S. Control of Markov chains with long-run average cost criterion. Stochastic Differential Systems, Stochastic Control Theory and Applications (Minneapolis, 1986). New York: Springer-Verlag, 1988, p. 57-77.

12. Borkar V.S. Control of Markov chains with long-run average cost criterion: The dynamic programming equations. - SIAM J. Control Optim., 1989, v. 27, № 3, p. 642657.

13. Carlson D. A., Haurie A. B., Leizarowitz A. Infinite Horizon Optimal Control: Deterministic and Stochastic Systems. Berlin: Springer-Verlag, 1991, 332 p.

14. Chen H. F., Guo L. Optimal adaptive control and consistent parameter estimates for ARMAX-model with quadratic cost. - SIAM J. Control Optim., 1987, v. 25, № 4, p. 845-867.

15. Chow Y.S., Teicher H. Probability Theory: Independence, Interchangeability, Martingales. New York: Springer-Verlag, 1997, 488 p.

16. Di Masi G. B., Kabanov Yu.M. On sensitive probabilistic criteria in the linear regulator problem with the infinite horizon. - Обозрение прикл. и промышл. матем., 1998 , т. 5 , B. 2 , с. $410-422$.

17. Евстигнеев И. В. Регулярные условные математические ожидания случайных величин, зависящих от параметров. - Теория вероятн. и ее примен., 1986, т. 31, в. 3 , с. $586-589$.

18. Флеминг У., Ришел $Р$. Оптимальное управление детерминированными и стохастическими системами. М.: Мир, 1978, 316 с.

19. Гихман И. И., Скороход А. В. Управляемые случайные процессы. Киев: Наукова думка, 1977, $251 \mathrm{c}$.

20. Hall P., Heyde C. C. Martingale Limit Theory and its Application. New York-London: Academic Press, 1980, 308 p.

21. Hernández-Lerma O., Lasserre J. B. Further Topics on Discrete-time Markov Control Processes. New York: Springer-Verlag, 1999, 276 p.

22. Hinderer $K$. Foundations of non-stationary dynamic programming with discrete time parameter. Berlin-New York: Springer-Verlag, 1970, 160 p.

23. Ватанабэ С., Икэда $H$. Стохастические дифференциальные уравнения и диффузионные процессы. М.: Наука, 1986, 445 с.

24. Karatzas I., Shreve S.I. Brownian Motion and Stochastic Calculus. New York: Springer-Verlag, 1991, $470 \mathrm{p}$.

25. Kertz R.P. Renewal plans and persistent optimality in countably additive gambling. - Math. Oper. Res., 1982, v. 7, № 3, p. 361-382.

26. Квакернаак $X$., Сиван $P$. Линейные оптимальные системы управления. М.: Мир, $1977,625 \mathrm{c}$.

27. Leizarowitz $A$. Infinite horizon stochastic regulation and tracking with the overtaking criterion. - Stochastics, 1987, v. 22, №2, p. 85-110. 
28. Leizarowitz $A$. On almost sure optimization for stochastic control systems. - Stochastics, 1988 , v. 23 , № 2, p. 85-107.

29. Leizarowitz A. Overtaking and almost sure optimality for infinite horizon Markov decision processes. - Math. Oper. Res., 1996, v. 21, № 1, p. 158-181.

30. Lippman $S$. Maximal average-reward controls for semi-Markov decision processes with arbitrary space and action space. - Ann. Math. Statist., 1971, v. 42, p. 1717-1726.

31. Липчер P. Ш., Ширяев А. Н. Теория мартингалов. М.: Наука, 1986, 512 с.

32. Mandl P. Estimation and control in Markov chains. - Adv. Appl. Probab., 1974, v. 6 , № 1 , p. $40-60$.

33. Mandl $P$. The use of optimal stationary policies in the adaptive contol linear systems. - Proceedings of the Symposium to honour J. Neymann (Warsaw, 1974). Warsaw: PWN, 1977, p. 223-242.

34. Mandl $P$. Some results in the adaptive control of linear systems. - Transactions of the Seventh Prague Conference on Information Theory, Statistical Decision Functions, Random Processes and of the Eight European Meeting of Statisticians (Prague, 1974). Vol. A. Ed. by J. Kozesnic. Dordrecht: Reidel, 1977, p. 399-410.

35. Mandl $P$. On the adaptive control of countable Markov chains. - Probability Theory (Warsaw, 1976) Warsaw: PWN, 1979, p. 159-173.

36. Mandl $P$. Asymptotic ordering of probability distributions for linear controlled systems with quadratic cost. - Lecture Notes Control Inform. Sci., 1986, v. 78, p. 277283.

37. Mandl P., Ayllón M.R.R. On controlled Markov processes with an average cost criterion. - Kybernetika (Prague), 1987, v. 23, № 6, p. 433-442.

38. Presman E., Rotar' V., Taksar M. Optimality in probability and almost surely. The general scheme and a linear regulator problem. - Stochastics Stochastics Rep., 1993, v. 43, № 3-4, p. 127-137.

39. Пресман Э. Л. Оптимальность почти наверное и по вероятности для стохастического линейно-квадратического регулятора. - Теория вероятн. и ее примен., 1997, т. 42 , в. 3 , с. $627-632$.

40. Ротарь В.И. О достаточных управлениях в динамической схеме стохастической оптимизации. - Матем. заметки, 1986, т. 40, № 4, с. 542-551.

41. Ротарь В.И. Некоторые замечания об асимптотической оптимальности. - Исследования по вероятностным проблемам управления экономическими процессами. М.: ЦЭМИ РАН, 1986, с. 93-116.

42. Rotar' V. I. Connectivity property and optimality almost surely and in probability. New Trends in Probability and Statistics (Bakuriani, 1980). Vol. 1. Ed. by V. Sazonov and T. Shervashidze. Utrecht/Vilnius: VSP/Mokslas, 1991, p. 528-539.

43. Rotar' V. I. Probability Theory. River Edge, NJ: World Scientific, 1997, 414 c.

44. Shwartz A., Makowski A.M. Comparing policies in Markov decision processes: Mandl's lemma revisited. - Math. Oper. Res., 1990, v. 15, № 1, p. 155-174.

45. Юшкевич A. А., Читашвили Р. Я. Управляемые случайные последовательности и марковские цепи. - Успехи матем. наук, 1982, т. 37, № 6, с. 213-242.

Поступила в редакцию 8.IV.2003 